\title{
IMAGING STRUCTURAL PROPERTIES OF SOFT TISSUES USING ULTRASOUND
}

\author{
Timothy J. Hall, Michael F. Insana, James L. Fishback*, and Stanton J. Rosenthal \\ Department of Diagnostic Radiology \\ *Department of Pathology \\ University of Kansas Medical Center \\ Kansas City, Kansas 66103
}

\begin{abstract}
We are developing methods to estimate and image structural properties of soft tissues. The basis for our methods are measurements of acoustic backscatter coefficients. From the backscatter coefficients four parameters are estimated and displayed in images. The ability to image independent physical properties of the tissue provides more descriptive information than a B-mode image which combines these properties.
\end{abstract}

\section{INTRODUCTION}

The goal of ultrasound tissue characterization is to more effectively use the information contained in the echo signals backscattered from tissues. Parameters used in tissue characterization should quantitatively describe the physical properties of the scattering structures and be sensitive to the changes in tissue microstructure that accompany disease processes.

The acoustic backscatter coefficient is a property intrinsic to the scattering medium, and has been studied by several groups involved in ultrasound tissue characterization. The backscatter coefficient for a given medium depends on the size and shape of the scattering structures, their number density and impedance difference relative to their surroundings, and the frequency of the acoustic pulse used to probe the scattering medium. Thus, the backscatter coefficient combines the effects of several tissue parameters.

The objective of our current work is to use the acoustic backscatter coefficient as the basis for estimation of more fundamental parameters such as scatterer size and scattering strength. An important part of this work is to determine the accuracy with which these parameters can be estimated.

\section{METHODS}

\section{Theory}

The theoretical basis for our data analysis is described in detail in Ref.[1], and its application to parameteric imaging in Ref.[2]. The following is an overview of the analysis.

The backscatter coefficient for media containing discrete scatterers can be written as

$$
\sigma_{b}(f)=C f^{4} D^{6} \bar{n} \gamma_{0}^{2} F
$$

where $C$ is a constant, $f$ is frequency, $D$ is the average scatterer diameter, $\bar{n}$ is their number per unit volume, and $\gamma_{0}^{2}$ is their impedance difference relative to the surrounding media. The product $\bar{n} \gamma_{0}^{2}$ is referred to as the scattering strength. $F$ is the acoustic intensity form factor, which is the Fourier transform of the correlation function of the scattering medium. The form factor accounts for such parameters as the scatterer size, shape, orientation, relative position (random or periodic), and their elastic properties. With prior knowledge or independent measurement of these parameters, the form factor can be reduced to a function of only frequency and scatterer size, $F \Rightarrow F(f, D)$. In the long-wavelength limit $F(f, D) \simeq 1$ and Eq. 1 reduces to the backscatter coefficient for Rayleigh scattering, $\sigma_{0}$. Hence, the form factor can be represented as

$$
F(f, D)=\frac{\sigma_{b}}{\sigma_{0}} .
$$

With this formulation, any variation in the frequency dependence of $\sigma_{b}(f)$ from $f^{4}$ is attributed to scatterer sizes larger than Rayleigh scatterers.

Form factors are modeled using prior information about the geometric, elastic, and organizational properties of the scattering medium. For simple random media, such as glass-sphere-in-agar test samples, scattering theory [3] provides all the means necessary for accurately modeling form factors. For the case of scattering from tissues, where scattering structures are not well defined, a Gaussian model is used.

Form factors are measured by estimating backscatter coefficients over the signal bandwidth of the transducer, dividing the result by $f^{4}$ and rescaling the result such that the extrapolated value at zero frequency is one.

\section{Parameter Estimation}

From backscatter coefficient estimates four parameters are estimated, as described in Ref.[1,2]. The average scatterer size is estimated by analyzing the frequency dependence of the backscatter coefficients via the form factor, $F(f, D)$. A chi-square goodness-of-fit test is used to test the equivalence of model functions and data. Scatterer size estimates are determined on the basis of a minimum chisquare criterion. The same chi-square measure is used as a parameter to indicate locations where the model function used are inappropriate. The scattering strength, $\bar{n} \gamma_{0}^{2}$, is de-

Annual International Conference of the IEEE Engineering in Medicine and Biology Society, Vol. 12, No. 1, $1990 \quad 0327$ CH2936-3/90/0000-0327\$01.00 @ 1990 IEEE 
termined by the magnitude of the backscatter coefficient via Eq. 1. Finally, the integrated backscatter coefficient (IBC) is computed over the signal bandwidth. IBC estimates have a lower statistical uncertainty than the backscatter coefficient estimates at any particular frequency, resulting in relatively low noise parametric images.

It is important to display the parameter estimates in an image format to preserve the spatial context of the information. Spatial resolution is acheived at the expense of parameter uncertainty. This is often a desirable trade-off because it takes advantage of the ability of the eye-brain system to distinguish among subtle variations in image parameters.

\section{RESULTS}

\section{Phantom Studies}

Phantoms were used to verify the accuracy of parameter estimates under controlled conditions with known scattering media. The phantoms consisted of microscopic spheres randomly distributed in an agar.

For a variety of phantoms containing discrete scatterers with one average scatterer size in a target area and another in the surrounding background, we have estimated scatterer sizes to within 10-20 percent in both target and background areas. As a result, image contrast was increased in scatterer size and strength images over B-mode images such that image contrast nearly equaled object contrast. For these phantoms, where the scatterers have similar composition but different size, the chi-square values in both the target and background are uniform, indicating the form factor model is appropriate for the entire phantom.

In other situations, the accuracy of scatterer size estimates is lost. For example, at very high frequencies the simple form factor models used become inadequate and the solution for scatterer sizes is not unique. Often the parametric image will have improved target detectibility over a $\mathrm{B}$-mode image, but parametric image interpretation is more difficult.

When using an accurate form factor model, we found that increasing the pulse bandwidth reduced the variance of the estimate without significantly changing measurement bias. The result is a decrease in parametric image noise as the pulse bandwidth is increased, improving the overall signal-to-noise ratio for low-contrast targets. Very broad bandwidth pulses combined with more detailed model functions can reduce the difficulties encountered at high frequencies.

\section{Tissue Studies}

We have begun parametric imaging of fresh dog kidneys in vitro. Ultrasonic parameter estimates in the cortex, medulla, and pelvis show some variation in scatterer size estimates and chi-square values, but large variations in the scattering strength and IBC values. Parametric image variations correspond to changes seen visually.
Scatterer size estimates at $7 \mathrm{MHz}$ in the cortex are uniform and in good agreement with published values for the average glomerulus diameter in dog kidneys: roughly $90-100 \mu \mathrm{m}$. Thus spatial variations in backscatter coefficients indicate changes in the number of scatterers and impedance difference in these regions. It also suggests that the glomerulus is an important scattering structure in the kidney cortex.

In medullary tissues chi-square values are greater than in the cortex. The higher chi-square values indicate that the spherically-symmetric form factor models used are not appropriate in these regions. In fact, the medulla consists of densely-packed tubules, structures with cylindrical symmetry. The kidney is one example where parametric imaging leads to more detailed information regardiffg the internal structure.

\section{CONCLUSIONS}

A method for estimation of fundamental acoustic properties has been developed and tested using well-characterized scattering media. Application of these parametric imaging techniques to phantoms showed that parameter estimations can be accurate and increased low-contrast target detectibility is possible.

Application of these techniques to parametric imaging of tissues provides images indicative of the spatial variations in tissue type viewed macroscopically, and provides more descriptive information than provided by B-mode imaging.

An important extension to this work will be to combine the individual parameter images into one tissue type image. Cross correlation values suggest that there is only a weak correlation among these parameters. Therefore, combining parameters via standard pattern recognition techniques might allow formation of a single tissue type image based on these parameters.

This work was supported in part by the West Trust and the Whitaker Foundation.

\section{REFERENCES}

[1] MF Insana, RF Wagner, DG Brown, and TJ Hall, "Describing small-scale structure in random media using pulse-echo ultrasound," J. Acoust. Soc. Am. 81(1), 179-192 (1990)

[2] MF Insana and TJ Hall, "Parametric ultrasound imaging from backscatter coefficient measuremnts: Image formation and interpretation," submitted for publication in Ultrasonic Imaging (Feb. 1990)

[3] JJ Faran, "Sound scattering by solid cylinder and shperes," J. Acoust. Soc. Am. 23, 405-418 (1951)

Send all correspondence to T.J. Hall at the address shown above or call 913-588-7868. 